INPLASY

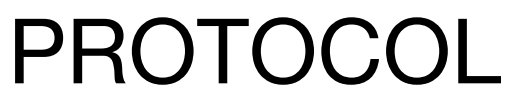

To cite: Guo. Alveolar bone changes in maxillary and mandibular anterior teeth during orthodontic treatment: A systematic review and metaanalysis. Inplasy protocol 202040174. doi: 10.37766/inplasy2020.4.0174

Received: 25 April 2020

Published: 25 April 2020

Corresponding author: Runzhi Guo

runzhiguo2017@163.com

Author Affiliation:

Peking University

Support: None

Review Stage at time of this submission: Formal screening of search results against eligibility criteria.

Conflicts of interest: None to declare.

\section{Alveolar bone changes in maxillary and mandibular anterior teeth during orthodontic treatment: A systematic review and meta-analysis}

Guo RZ.

Review question / Objective: This systematic review and meta-analysis analyzed the effect of tooth movement on alveolar bone changes, as measured by CBCT, of both maxillary and mandibular anterior teeth.

Condition being studied: The association between tooth movement and remodeling of surrounding bone is an important research topic. However, the relationship between anterior tooth movement and alveolar bone changes remains unclear.

Information sources: The electronic search of Embase, Cochrane Library, and Medline (via PubMed) databases. Manual screening of three major orthodontic journals (European Journal of Orthodontics, American Journal of Orthodontics and Dentofacial Orthopedics, and Angle Orthodontist), and the reference lists of the selected articles were manually searched for additional studies.

INPLASY registration number: This protocol was registered with the International Platform of Registered Systematic Review and Meta-Analysis Protocols (INPLASY) on 25 April 2020 and was last updated on 25 April 2020 (registration number INPLASY202040174).

\section{INTRODUCTION}

Review question / Objective: This systematic review and meta-analysis analyzed the effect of tooth movement on alveolar bone changes, as measured by CBCT, of both maxillary and mandibular anterior teeth.
Rationale: At present, the bone remodeling during orthodontic treatment is still unknown. To avoid iatrogenic bone loss during orthodontic treatment, understanding the bone remodeling ability and establishing the amount of tooth movement prior to orthodontic treatment is important. 
Condition being studied: The association between tooth movement and remodeling of surrounding bone is an important research topic. However, the relationship between anterior tooth movement and alveolar bone changes remains unclear.

\section{METHODS}

Participant or population: Orthodontic patients with permanent dentition, treated with a fixed appliance, were included.

Intervention: The included patients were treated with a full-mouth fixed appliances with brackets.

Comparator: The untreated control group with at least two exposures to CBCT radiation was included, where available.

Study designs to be included: Longitudinal studies, both retrospective and prospective, using СВСТ to observe alveolar bone changes in maxillary and mandibular anterior teeth durin.

Eligibility criteria: Longitudinal studies using СВСТ to observe alveolar bone changes of maxillary and mandibular anterior teeth during orthodontic treatment were included.

Information sources: The electronic search of Embase, Cochrane Library, and Medline (via PubMed) databases. Manual screening of three major orthodontic journals (European Journal of Orthodontics, American Journal of Orthodontics and Dentofacial Orthopedics, and Angle Orthodontist), and the reference lists of the selected articles were manually searched for additional studies.

Main outcome(s): Changes in alveolar bone measurements in the anterior region of the maxilla and mandible, as analyzed by CBCT, were the outcome measures.

Quality assessment / Risk of bias analysis: For randomized clinical trials, the risk of bias tool of the Cochrane Collaboration was used to evaluate the risk of bias. The Methodological Index for Non-randomized
Studies (MINORS) was used to evaluate the methodological quality of non-randomized clinical studies. The overall quality of evidence for outcome was assessed using the Grading of Recommendations Assessment, Development and Evaluation (GRADE) approach.

Strategy of data synthesis: A meta-analysis was conducted on the quantitative data using Review Manager 5.3 (Nordic Cochrane Centre, Cochrane Collaboration, Copenhagen, Denmark). Taking in consideration the high clinical heterogeneity among the included studies, the random effect model was used for the analysis. The statistical heterogeneity was assessed using the chi-square and Isquare tests. The 12 value of $25 \%, 50 \%$ and $75 \%$ were indicated as low, moderate and high heterogeneity. P-values $\leq 0.05$ were considered statistically significant. Studies with methods and outcomes that could not be quantitatively analyzed were described qualitatively.

Subgroup analysis: The subgroup analysis was performed to reduce the heterogeneity from different treatment duration, skeletal type, tooth movement direction and periodontal status.

Sensibility analysis: Sensitivity analysis was conducted to test the stability of some of the debatable results in the meta-analysis.

Country(ies) involved: China.

Keywords: Orthodontic, Alveolar bone, CBCT. 